\title{
Psicooncología
}

ISSN: $1696-7240$

\section{Religiosidad, espiritualidad y calidad de vida en familiares de pacientes de oncología pediatrica en un hospital de referencia en el noreste de Brasil}

\author{
Amanda Rebeca Torres Furtado de Mendonça ${ }^{1 *}$, Rodolpho Omena Cabral ${ }^{2}$, Arturo de Padua \\ Walfrido Jordan ${ }^{3}$, Alberto Gorayeb de Carvalho Ferreira ${ }^{4}$, Leopoldo Nelson Fernandes \\ Barbosa $^{5}$
}

Recibido: 1 de junio de 2020 / 10 de agosto de 2020

Resumen: Objetivo: caracterizar el perfil sociodemográfico y analizar la asociación entre religiosidad, espiritualidad, como elementos para enfrentar la enfermedad y calidad de vida en familiares de pacientes de oncología pediátrica de hospital de referencia. Método: estudio transversal descriptivo con metodología cuantitativa, realizado de 2017 a 2018. Datos fueron recolectados a través de Whoqol bref y la Escala de Afrontamiento Religioso/Espiritual Breve. Resultados: se entrevistó a 47 familiares, con perfil compuesto predominantemente por parientes femeninos, de 30 a 39 años, casados, madres con más de 1 hijo, residentes del interior del estado, con educación primaria incompleta, ingresos familiares de 1 a 3 salarios mínimos y religión protestante. Se observó que los ancianos y los que abandonaron su hogar para buscar mejores tratamientos tenían el peor índice de calidad de vida, mientras que las personas casadas y los jóvenes tenían la mejor. Las personas espiritualizadas demostraron ser más capaces de afrontar las adversidades, así como aquellas cuya frecuencia religiosa aparece regularmente. También se notó que la mayoría afirma que la religiosidad y espiritualidad son muy importantes, pero a pocas se les habló sobre ellas durante la atención médica. Conclusiones: para el público evaluado, la espiritualidad es un factor importante para hacer frente a situaciones estresantes, así como para proporcionar mejor calidad de vida a los familiares. Científicamente, la necesidad de más investigación sobre el tema es evidente, dado que el valor de las futuras contribuciones al campo de la salud es indudable.

Palabras clave: Familia, oncología médica, calidad de vida, religión, espiritualidad, adaptación psicológica, psicooncología.

1 Amanda Rebeca Torres Furtado de Mendonça Faculdade Pernambucana de Saúde. Estado de Pernambuco Brasil

E-mail: amandarebeca@hotmail.com

2 Rodolpho Omena Cabra. Faculdade Pernambucana de Saúde. Estado de Pernambuco - Brasil

E-mail: rodolphoomena@gmail.com

3 Arturo de Padua Walfrido Jordan Faculdade Pernambucana de Saúde. Estado de Pernambuco - Brasil

E-mail: arturojor@yahoo.com.br

4 Alberto Gorayeb de Carvalho Ferreira. Faculdade Pernambucana de Saúde. Estado de Pernambuco - Brasil E-mail: gorayeb.alberto@gmail.com

5 Leopoldo Nelson Fernandes Barbosa. Faculdade Pernambucana de Saúde. Estado de Pernambuco - Brasil E-mail: leopoldopsi@gmail.com

* Dirección de correspondencia: Amanda Rebeca Torres Furtado de Mendonça. Faculdade Pernambucana de Saúde; Avenida Mal. Mascarenhas de Morais, 4861, barrio Imbiribeira, ciudad de Recife, estado de Pernambuco, código postal: 51.150-000; E-mail: amandarebeca@hotmail.com 


\title{
[en] Religiosity, spirituality and quality of life in family members of pediatric oncology patients of a referral hospital in the northeastern brazil
}

\begin{abstract}
Objective: to characterize the sociodemographic profile and analyze the association between religiosity, spirituality, as elements to face the sickness and quality of life in relatives of pediatric oncology patients at a referral hospital. Method: descriptive cross-sectional study with quantitative methodology, carried out from 2017 to 2018. Data collected through Whoqol bref and Brief Spiritual/ Religious Afrontamiento Scale. Results: 47 family members were interviewed, with a profile composed predominantly of female relatives, from 30 to 39 years old, married, mothers with more than 1 child, upstate residents, with incomplete primary education, family income of 1 to 3 minimum wages and protestant religion. It was noted that the elderly and those who abandoned their home to seek the best treatment had the worst average for quality of life, while married people and young people had the best. Spiritualized people have shown themselves to be more able to cope with adversity, as those whose religious frequency appears regularly. Moreover, the majority affirms that religiosity and spirituality are very important, but few were approached spiritually during medical care. Conclusion: in relation to the evaluated public, spirituality is an important factor when dealing with stressful situations, as well as to provide better quality of life for family members. Scientifically, the need for more research on the topic is evident, given the indisputable value of future contributions to the field of health.
\end{abstract}

Keywords: Family, medical oncology, quality of life, religion, spirituality, adaptation, psycho-oncology.

Sumario: 1. Introducción 2. Método 3. Resultados 4. Discusión 5. Conclusiones 6. Referencias bibliográficas

Cómo citar: Torres Furtado de Mendonça AR, Omena Cabral R, Walfrido Jordan AP, Gorayeb de Carvalho Ferreira A, Fernandes Barbosa LN. Religiosidad, espiritualidad y calidad de vida en familiares de pacientes de oncología pediatrica en un hospital de referencia en el noreste de Brasil. Psicooncología 2020; 17: 273-291. doi: 10.5209/psic.69778

\section{Introducción}

El tema de la religiosidad/espiritualidad (R/E) está ganando cada vez más importancia en el área de la medicina tradicional. Las experiencias de auto trascendencia son aspectos tan frecuentes y inspiradores de la vida humana que sería impensable comprender su naturaleza sin investigar estos fenómenos ${ }^{(1)}$. De esa forma, hoy en día, hay poco espacio para preguntas sobre la importancia de $\mathrm{R} / \mathrm{E}$ en la atención al paciente, aunque todavía hay dudas sobre cómo debería ser este apoyo espiritual ${ }^{(1,2)}$.

Entre las diversas afecciones médicas que pueden estar estrechamente vinculadas con el tema de la $\mathrm{R} / \mathrm{E}$ se encuentra el cáncer infanti ${ }^{(3,4)}$. A pesar del amplio progreso en el tratamiento del cáncer, la enfermedad implica un tratamiento prolongado que exige cuidados y necesidades especiales, los cuales pueden conducir a un estado de depresión, aislamiento e incluso desesperanza ${ }^{(5)}$. Para hacer frente a esta situación, los pacientes con cáncer y sus familiares utilizan diferentes estrategias de afrontamiento, siendo R/E una de ellas ${ }^{(4)}$.

El afrontamiento se define como los esfuerzos cognitivos y conductuales dirigidos para el manejo de las demandas, evaluadas como una sobrecarga de recursos personales. Por lo tanto, muchos eligen estrategias de afrontamiento para hacer frente a las adversidades de la vida, como diagnóstico y tratamiento del cáncer ${ }^{(3,6)}$.

En la práctica del cáncer pediátrico, el bienestar espiritual de los pacientes y sus familiares deben ocupar un espacio relevante en la relación médico-paciente/ 
familia. En este aspecto, la dimensión espiritual y religiosa se destaca como parte integral de la atención ${ }^{(7,8)}$.

Existen numerosas definiciones en la literatura sobre religiosidad y espiritualidad. La religión es un sistema organizado que está relacionado con la fuerza divina o sobrenatural; está vinculado a lo sagrado y a una doctrina; sirve como el vehículo por el cual el individuo expresa su espiritualidad ${ }^{(1,2,9)}$. La religiosidad, a su vez, es lo que un individuo cree, sigue y practica una religión ${ }^{(3,10)}$.

La espiritualidad, por otro lado, implica un concepto más amplio que la religión, siendo definido como la búsqueda personal para comprender las respuestas a las preguntas de la vida, su significado y relaciones con lo sagrado y trascendente, que pueden o no estar relacionadas con propuestas de una religión en particular ${ }^{(11)}$.

En este contexto, también es interesante comprender la salud y la calidad de vida $(\mathrm{CV})$, ya que este último es una medida importante de impacto sobre la promoción de la primera. Según la Organización Mundial de la Salud (OMS), el concepto de salud abarca no solo la ausencia de enfermedad, pero comprende una situación de completo bienestar físico, mental y social. Cuando se vincula con la CV, implica la percepción del ser sobre su posición en el mundo según el contexto cultural. Es el sistema de valores con los cuales el ser convive en relación con sus objetivos, estándares, preocupaciones y expectativas ${ }^{(9)}$.

Con respecto a los pacientes con cáncer pediátrico y sus familiares, los estudios indican que la CV relacionada con la salud percibida por los padres es peor que la percibida por los niños. Así, como elemento fundamental del equipo de salud en la recuperación del paciente, los padres deben ser objeto de intervenciones de reducción de la ansiedad por su participación eficaz y eficiente en la atención ${ }^{(3,4,12)}$.

Un ámbito importante de estrategia de afrontamiento utilizadas por las personas para adaptarse a circunstancias adversas o estresantes en la vida es el afrontamiento religioso/espiritual (CRE). El CRE puede ser positivo ou negativo, conforme esté asociado con indicadores de mejor o peor salud. Cuando es positivo (CREP) está relacionado con efectos beneficiosos para los humanos, como buscar el amor y proteger a las deidades. Ya el CRE negativo (CREN) cubre las consecuencias que son perjudiciales para el individuo, por ejemplo, redefinir los factores estresantes como castigo divino ${ }^{(1,2,6)}$.

Recientemente, los estudios en $\mathrm{R} / \mathrm{E}$ han demostrado asociaciones favorables relacionadas con la salud, incluyendo una mejor salud mental y $\mathrm{CV}$, mayor sobrevida, reducción del dolor y prevalencia de la enfermedad en pacientes con cáncer ${ }^{(1,2,4)}$. Con menos frecuencia, algunos estudios también indican la existencia de peores resultados, asociado principalmente con pensamientos punitivos, culpa, intolerancia $\mathrm{y}$ abandono de tratamientos médicos ${ }^{(1,2,10)}$.

No obstante, aunque todos los aspectos listado anteriormente son dignos de atención, todavía hay escasez de publicaciones que vinculen la influencia de la religiosidad y la espiritualidad en el cuidado de los familiares de pacientes pediátricos con cáncer, lo que hace que esta investigación sea relevante ya que puede contribuir a mejorar la CV de los involucrados, así como la asistencia ofrecida.

Además, los estudios indican que los familiares, especialmente los padres, son los más afectados por el diagnóstico de cáncer del niño, haciéndolos, por tanto, componentes determinantes de las intervenciones del equipo de salud para reducir la ansiedad, con la intención de efectuar participación en la atención, buscando siempre la recuperación y el bienestar del paciente ${ }^{(3,4,12)}$. 
En esta perspectiva, este estudio tiene como objetivo caracterizar el perfil sociodemográfico y analizar la asociación entre religiosidad, espiritualidad, como elementos de afrontamiento a la enfermedad, y CV en familiares de pacientes pediátricos oncológicos del Instituto de Medicina Integral Profesor Fernando Figueira (IMIP).

\section{Método}

La población de estudio consistió en 47 familiares de pacientes con cáncer pediátrico ingresados en el IMIP, siendo un familiar el interrogado por cada niño enfermo. Para su inclusión en el estudio, era necesario ser familiar del paciente con cáncer pediátrico de hasta 13 años de edad (límite de edad de pediatría en la institución) en tratamiento en la sala de observación del IMIP. Fueron excluidos de la muestra los acompañantes sin grado de parentesco con los pacientes y aquellos que no completaron los instrumentos de colecta en su totalidad.

Se realizó un estudio descriptivo de corte transversal con metodología cuantitativa.

Para la investigación, el cuestionario se compuso de preguntas cerradas sobre características sociodemográficas, académicas y profesionales. Además, se adjuntaron al cuestionario la Escala de Afrontamiento Religioso/Espiritual Breve (CRE-Breve) y el Whoqol-bref de la OMS.

CRE-Breve es la forma reducida de la Escala de Afrontamiento Religioso/ Espiritual, que sirve como un instrumento para comprender cómo la $\mathrm{R} / \mathrm{E}$ puede actuar ante la adversidad. La escala contiene 49 ítems divididos en dos dimensiones, una positiva con 34 ítems y una negativa con 15 ítems $^{(7,13)}$. El Whoqol-bref consta de 26 preguntas y las respuestas siguen una escala de 1 a 5, cuanto mayor sea el puntaje, mejor será la $\mathrm{CV}^{(14)}$.

La recopilación de datos tuvo lugar durante los meses de enero a abril de 2018 , durante los cuales se invitó a los miembros de la familia a participar en la entrevista después de ser informados sobre los objetivos de la investigación y de firmar el Formulario de consentimiento informado (ICF).

Los datos recopilados fueron almacenados y organizados en una hoja de cálculo de Excel. Las comparaciones de medias entre dos grupos o entre dos ocasiones en el mismo grupo, fueron efectuadas, respectivamente, con la prueba $t$ de Student para muestras independientes y muestras pareadas. Los datos categóricos se resumieron a través de proporciones y las comparaciones de estos intragrupos se realizaron mediante la prueba de chi-cuadrado de Pearson o la prueba exacta de Fisher, cuando justificado. En el análisis de correlación se utilizó el coeficiente de correlación de Pearson. En todos los tanteos, se adoptó el nivel de significancia del 5\%. El software utilizado en el análisis estadístico fue R.

Este estudio fue aprobado por el Comité de Ética en Investigación Humana del IMIP y siguió todos los parámetros de las resoluciones 466/12 e 510/16 de la Comisión Nacional de Ética de Investigación (CONEP).

\section{Resultados}

De los 47 sujetos entrevistados, la mayoría son mujeres, madres de los pacientes hospitalizados, quienes tienen más de un hijo. El rango de edad es de 30 a 39 años 
y la edad promedio es de 35 años; son casadas, residentes del interior de Pernambuco, con educación primaria incompleta; poseen trabajo, tienen un ingreso familiar de 1 a 3 salarios mínimos, tienen religión, se dedican una o más veces a la religión por semana y afirman que la religiosidad y la espiritualidad son muy importantes (tabla 1).

Tabla 1. Características sociodemográficas y personales de los familiares de pacientes hospitalizados en la sala de observación de oncología pediátrica del IMIP.

\begin{tabular}{lccc}
\hline & & N & \% \\
\hline Sexo & & & \\
& Masculino & 7 & 14,9 \\
& Femenino & 40 & 85,1 \\
\hline
\end{tabular}

\section{Edad}

$\begin{array}{rcc}17-29 \text { años } & 14 & 29,8 \\ 30-39 \text { años } & 21 & 44,7 \\ 40-49 \text { años } & 8 & 17,0 \\ \geq 50 \text { años } & 4 & 8,5\end{array}$

\section{Ciudad}

$\begin{array}{rcc}\text { Recife } & 10 & 21,3 \\ \text { Región Metropolitana de Recife } & 12 & 25,5 \\ \text { Interior de Pernambuco } & 20 & 42,6 \\ \text { Otros estados } & 5 & 10,6\end{array}$

\section{Nivel Académico}

$\begin{array}{rcc}\text { Educación Primaria Incompleta } & 19 & 40,4 \\ \text { Educación Primaria Completa } & 4 & 8,5 \\ \text { Educación Secundaria Incompleta } & 2 & 4,3 \\ \text { Educación Secundaria Completa } & 15 & 31,9 \\ \text { Educación Superior Incompleta } & 2 & 4,3 \\ \text { Educación Superior Completa } & 4 & 8,5 \\ \text { Posgraduación } & 1 & 2,1\end{array}$

\section{Actividad laboral}

$\begin{array}{rrr}\text { Sí } & 33 & 70,2 \\ \text { No } & 14 & 29,8\end{array}$




\section{Ingresos familiares}

$\begin{array}{rcc}\text { Menos de 1 salario mínimo } & 11 & 23,4 \\ \text { 1 a } 3 \text { salarios mínimos } & 34 & 72,3 \\ \text { 4 a } 7 \text { salarios mínimos } & 2 & 4,3\end{array}$

\section{Estado Civil}

$\begin{array}{rcc}\text { Soltero } & 5 & 10,6 \\ \text { Casado } & 21 & 44,7 \\ \text { Separado } & 7 & 14,9 \\ \text { Relación estable } & 14 & 29,8\end{array}$

\section{Parentesco con el paciente en tratamiento}

$\begin{array}{rcc}\text { Padre } & 6 & 12,8 \\ \text { Madre } & 33 & 70,2 \\ \text { Tío/Tía } & 3 & 6,4 \\ \text { Hermano/Hermana } & 2 & 4,2 \\ \text { Abuelo/Abuela } & 3 & 6,4\end{array}$

Padre/madre con más de un hijo

$\begin{array}{rrr}\text { Sí } & 29 & 74,4 \\ \text { No } & 10 & 25,6\end{array}$

\section{Religión}

$\begin{array}{rcc}\text { Católico } & 18 & 38,3 \\ \text { Protestante } & 20 & 42,6 \\ \text { cree en Dios } & 9 & 19,1\end{array}$

\section{Frecuencia dedicada a las prácticas religiosas}

$\begin{array}{rcc}\geq 1 \text { vez por semana } & 30 & 63,8 \\ 1 \text { a } 2 \text { veces por mes } & 9 & 19,1 \\ \text { Algunas veces al año } & 3 & 6,4 \\ \text { Raramente } & 5 & 10,6\end{array}$

\section{Importancia de la Religiosidad/Espiritualidad}

$\begin{array}{rcc}\text { Muy importante } & 40 & 85,1 \\ \text { Moderadamente importante } & 6 & 12,8 \\ \text { Poco importante } & 1 & 2,1\end{array}$


También se observó que la mayor parte de los niños habían sido diagnosticados con cáncer hace menos de 6 meses y el sumo de los miembros de la familia creen que, a lo largo del proceso, el principal factor estresante fue la aceptación/diagnóstico de la enfermedad. Además, la mayoría de los encuestados declaran que su propia R/E nunca fue abordado por profesionales de la salud durante los períodos de consulta y hospitalización, también atestiguan que la $\mathrm{R} / \mathrm{E}$ del paciente no fue abordado por los mismos profesionales y aseguran que el enfoque de R/E durante estos tiempos es muy importante (tabla 2).

Tabla 2. Características epidemiológicas de los familiares de pacientes hospitalizados en la sala de observación de oncología pediátrica del IMIP.

\begin{tabular}{lrcc}
\hline & $\mathbf{N}$ & $\%$ \\
\hline $\begin{array}{l}\text { Tiempo transcurrido desde que el niño fue diagnostica- } \\
\text { do con cáncer }\end{array}$ & & \\
& 0 a 6 meses & 26 & 55,3 \\
$>$ 6 meses a 1 año & 5 & 10,7 \\
$>1$ año a 1 año e 6 meses & 4 & 8,5 \\
$>1$ año e 6 meses a 2 años & 4 & 8,5 \\
$>2$ años & 8 & 17,0
\end{tabular}

\section{Mayor factor estresante durante todo el proceso de la} enfermedad

$\begin{array}{rcc}\text { Aceptación/diagnóstico } & 30 & 63,9 \\ \text { Internación } & 4 & 8,5 \\ \text { Tratamiento } & 11 & 23,4 \\ \text { Recurrencia del cáncer } & 1 & 2,1 \\ \text { Falta de apoyo } & 1 & 2,1\end{array}$

\section{Abordaje del profesional de la salud con respecto a la $\mathbf{R} / \mathbf{E}$ del familiar}

$\begin{array}{rll}\text { Fue abordado } & 15 & 32,0 \\ \text { No fue abordado } & 32 & 68,0\end{array}$

\begin{tabular}{|c|c|c|c|}
\hline \multicolumn{4}{|c|}{$\begin{array}{l}\text { Abordaje del profesional de la salud con respecto a la } \\
\mathrm{R} / \mathrm{E} \text { del paciente }\end{array}$} \\
\hline & Fue abordado & 17 & 36,2 \\
\hline & No fue abordado & 30 & 63,8 \\
\hline \multicolumn{4}{|c|}{$\begin{array}{l}\text { Opinión de los familiares sobre el abordaje de la } \mathrm{R} / \mathrm{E} \\
\text { durante las consultas }\end{array}$} \\
\hline & Muy importante & 38 & 80,9 \\
\hline & No es importante & 9 & 19,1 \\
\hline
\end{tabular}


Al comparar cada dominio de $\mathrm{CV}$ y la percepción de $\mathrm{CV}$ de los participantes con las variables que presentaron valores relevantes, se notó que el dominio físico ofreció mejores resultados para aquellos que viven en Recife, al igual para aquellos que tienen un ingreso familiar de 4 a 7 salarios mínimos; mientras que los individuos que viven en otro estado y que disponen de un ingreso familiar de 1 a 3 salarios mínimos tuvieron los peores promedios. Por otro lado, para el dominio psicológico, los promedios más elevados los obtuvieron las personas que raramente se dedican a la religión y también a los hermanos/hermanas de los pacientes, mientras que los peores se mantuvieron con aquellos que frecuentan prácticas religiosas algunas veces al año y para los abuelos/abuelas. Para el dominio de las relaciones sociales, las medias más altas presentadas fueron de padres y de aquellos que consideraron la recurrencia del cáncer como el principal estresor. Además, los abuelos/abuelas y aquellos cuyo estrés más profundo fue en la fase de hospitalización, indicaron los peores resultados. Con respecto al dominio ambiental, los mejores promedios se observaron en trabajadores. Todos estos dominios influyen en la percepción de la $\mathrm{CV}$, por lo que el mejor resultado se encuentra en jóvenes de 17 a 29 años, a la vez que las personas mayores de 50 años mostraron la peor media (Tabla 3).

Tabla 3. Asociación entre los dominios de calidad de vida y las características sociodemográficas y personales de los familiares de pacientes hospitalizados en la sala de observación de oncología pediátrica del IMIP.

\begin{tabular}{|c|c|c|c|c|c|c|c|c|c|c|}
\hline \multirow{2}{*}{ Variables } & \multicolumn{2}{|c|}{ Salud Física } & \multicolumn{2}{|c|}{$\begin{array}{c}\text { Salud } \\
\text { Psicológica }\end{array}$} & \multicolumn{2}{|c|}{$\begin{array}{l}\text { Relaciones } \\
\text { sociales }\end{array}$} & \multicolumn{2}{|c|}{ Ambiente } & \multicolumn{2}{|c|}{$\begin{array}{l}\text { Percepción de la } \\
\text { calidad de vida }\end{array}$} \\
\hline & Média & P-valor & Média & P-valor & Média & P-valor & Média & P-valor & Média & P-valor \\
\hline \multicolumn{11}{|l|}{ Edad } \\
\hline 17 a 29 años & 59,2 & 0,256 & 74,7 & 0,250 & 74,4 & 0,204 & 56,5 & 0,917 & 73,2 & $0,008^{*}$ \\
\hline 30 a 39 años & 55,1 & & 67,4 & & 82,9 & & 54,5 & & 71,4 & \\
\hline 40 a 49 años & 57,5 & & 63,5 & & 65,6 & & 50,4 & & 54,7 & \\
\hline$\geq 50$ años & 67,0 & & 60,4 & & 64,6 & & 56,2 & & 50,0 & \\
\hline \multicolumn{11}{|l|}{ Ciudad } \\
\hline Recife & 64,6 & $0,032 *$ & 75,8 & 0,206 & 84,2 & 0,093 & 61,5 & 0,163 & 70,0 & 0,075 \\
\hline $\begin{array}{r}\text { Región Me- } \\
\text { tropolitana de } \\
\text { Recife }\end{array}$ & 56,2 & & 64,6 & & 66,0 & & 44,3 & & 64,6 & \\
\hline $\begin{array}{r}\text { Interior de } \\
\text { Pernambuco } \\
\end{array}$ & 57,8 & & 68,7 & & 81,2 & & 57,4 & & 68,1 & \\
\hline Otros estados & 47,2 & & 60,8 & & 61,7 & & 53,7 & & 65,0 & \\
\hline
\end{tabular}

\section{Ingresos}

familiares

\begin{tabular}{|c|c|c|c|c|c|c|c|c|c|}
\hline $\begin{array}{r}<1 \text { salario } \\
\text { mínimo }\end{array}$ & 62,0 & $0,003^{*}$ & 75,4 & 0,163 & 84,8 & 0,195 & 57,4 & 0,100 & 63,6 \\
\hline $\begin{array}{r}1 \text { a } 3 \text { salarios } \\
\text { mínimos }\end{array}$ & 55,1 & & 65,8 & & 72,0 & & 52,0 & & 68,0 \\
\hline
\end{tabular}




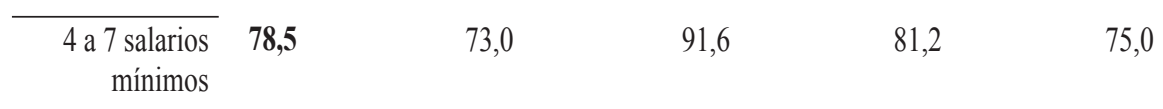

\section{Actividad}

laboral

$\begin{array}{rrrrrrrrrrr}\text { Sí } & 58,3 & 0,597 & 67,7 & 0,638 & 77,4 & 0,804 & \mathbf{5 8 , 2} & \mathbf{0 , 0 4 7 *} & 68,1 & 0,493 \\ \text { No } & 56,4 & & 70,0 & & 72,6 & & \mathbf{4 6 , 0} & & 62,0 & \end{array}$

\section{Frecuencia \\ dedicada a \\ las prácticas \\ religiosas}

\begin{tabular}{|c|c|c|c|c|c|c|c|c|c|c|}
\hline $\begin{array}{r}\geq 1 \text { vez por } \\
\text { semana }\end{array}$ & 56,6 & 0,555 & 69,3 & $0,001 *$ & 78,9 & 0,229 & 56,7 & 0,630 & 70,0 & 0,550 \\
\hline $\begin{array}{r}1 \text { a } 2 \text { veces por } \\
\text { mes }\end{array}$ & 60,7 & & 63,8 & & 73,1 & & 49,0 & & 61,1 & \\
\hline $\begin{array}{r}\text { Algunas veces } \\
\text { al año }\end{array}$ & 53,5 & & 45,8 & & 61,1 & & 45,8 & & 62,5 & \\
\hline Raramente & 62,1 & & 84,1 & & 71,6 & & 57,0 & & 65,0 & \\
\hline \multicolumn{11}{|l|}{$\begin{array}{l}\text { Parentesco con } \\
\text { el paciente }\end{array}$} \\
\hline Padre & 57,7 & 0,589 & 64,6 & $0,046^{*}$ & 83,3 & $0,041^{*}$ & 50,7 & 0,174 & 75,0 & 0,213 \\
\hline Madre & 58,7 & & 70,1 & & 80,0 & & 57,0 & & 67,8 & \\
\hline Tío/Tía & 53,6 & & 70,8 & & 66,6 & & 47,9 & & 58,3 & \\
\hline $\begin{array}{r}\text { Hermano/ } \\
\text { Hermana }\end{array}$ & 62,5 & & 81,2 & & 79,1 & & 70,3 & & 62,5 & \\
\hline Abuelo/Abuela & 48,8 & & 45,8 & & 22,2 & & 32,3 & & 58,3 & \\
\hline \multicolumn{11}{|l|}{$\begin{array}{l}\text { Mayor factor } \\
\text { estresante }\end{array}$} \\
\hline $\begin{array}{l}\text { Aceptación/ } \\
\text { diagnóstico }\end{array}$ & 60,0 & 0,125 & 69,2 & 0,334 & 81,6 & 0,039 * & 57,2 & 0,423 & 68,3 & 0,508 \\
\hline Internación & 50,0 & & 58,3 & & 52,1 & & 53,9 & & 53,1 & \\
\hline Tratamiento & 52,6 & & 67,0 & & 66,6 & & 46,6 & & 69,3 & \\
\hline $\begin{array}{r}\text { Recurrencia del } \\
\text { cáncer }\end{array}$ & 67,8 & & 65,0 & & 100,0 & & 46,9 & & 62,5 & \\
\hline Falta de apoyo & 67,8 & & 91,7 & & 75,0 & & 78,1 & & 75,0 & \\
\hline
\end{tabular}

En relación con los resultados obtenidos al comparar la escala CREP y sus dimensiones, se encontró que los sujetos que obtuvieron los mejores resultados presentan las siguientes características: educación superior incompleta (acciones en búsqueda del otro institucional) e ingresos familiares inferiores a 1 salario mínimo (acciones en búsqueda de ayuda espiritual); mientras que aquellos con escuela primaria incompleta tuvieron peores resultados. Se han mostrado también resultados 
más altos de "acciones que buscan ayuda espiritual" para aquellos cuyos ingresos son inferiores a 1 salario mínimo. En contraste, el resultado más bajo fue para aquellos con ingresos de 4 a 7 salarios mínimos. Con respecto a los padres de los pacientes, cuando no hay otro hijo, el promedio de "alejamiento a través de Dios, religión y/o espiritualidad" es mayor. En cuanto a la religión, el mejor resultado en la dimensión "acciones en búsqueda de ayuda espiritual" se encontró entre los católicos y el peor promedio entre los protestantes. Por último, la hospitalización alcanzó los resultados más altos en "transformación de sí y/o de su vida" y "acciones en búsqueda de ayuda espiritual" como el mayor estresante durante todo el proceso de la enfermedad; mientras que la recurrencia del cáncer y el tratamiento alcanzaron los promedios más bajos de "transformación de sí y/o de su vida" y "acciones en búsqueda de ayuda espiritual", respectivamente (Tabla 4).

Tabla 4. Asociación entre el CREP y sus dimensiones con las características sociodemográficas y personales de los familiares de pacientes hospitalizados en la sala de observación de oncología pediátrica del IMIP.

\begin{tabular}{|c|c|c|c|c|c|c|c|c|}
\hline \multirow[t]{2}{*}{ Variables } & \multicolumn{2}{|c|}{$\begin{array}{c}\text { Transformación } \\
\text { de sí y/o de su } \\
\text { vida }\end{array}$} & \multicolumn{2}{|c|}{$\begin{array}{c}\text { Acciones en } \\
\text { búsqueda de } \\
\text { ayuda espiritual }\end{array}$} & \multicolumn{2}{|c|}{$\begin{array}{c}\text { Acciones en } \\
\text { búsqueda del } \\
\text { otro institucional }\end{array}$} & \multicolumn{2}{|c|}{$\begin{array}{l}\text { Alejamiento a } \\
\text { través de Dios, } \\
\text { religión y/o } \\
\text { espiritualidades }\end{array}$} \\
\hline & Média & P-valor & Média & P-valor & Média & P-valor & Média & P-valor \\
\hline Nivel Académico & & 0,483 & & 0,709 & & 0,001 & & 0,861 \\
\hline $\begin{array}{r}\text { Primaria } \\
\text { Incompleta }\end{array}$ & 3,5 & & 1,9 & & 2,4 & & 4,2 & \\
\hline $\begin{array}{r}\text { Primaria } \\
\text { Completa }\end{array}$ & 4,0 & & 2,3 & & 3,7 & & 4,6 & \\
\hline $\begin{array}{l}\text { Secundaria } \\
\text { Incompleta }\end{array}$ & 4,5 & & 2,1 & & 2,5 & & 4,5 & \\
\hline $\begin{array}{r}\text { Secundaria } \\
\text { Completa }\end{array}$ & 3,5 & & 1,7 & & 2,5 & & 4,2 & \\
\hline $\begin{array}{r}\text { Superior } \\
\text { Incompleta }\end{array}$ & 3,7 & & 1,7 & & 4,1 & & 4,2 & \\
\hline $\begin{array}{r}\text { Superior } \\
\text { Completa }\end{array}$ & 3,8 & & 1,6 & & 3,5 & & 4,5 & \\
\hline Posgraduación & 3,0 & & 2,6 & & 3,3 & & 5,0 & \\
\hline $\begin{array}{l}\text { Ingresos } \\
\text { familiares }\end{array}$ & & 0,894 & & 0,009 & & 0,797 & & 0,827 \\
\hline $\begin{array}{r}<1 \text { salario } \\
\text { mínimo }\end{array}$ & 3,6 & & 2,5 & & 2,7 & & 4,3 & \\
\hline $\begin{array}{r}1 \text { a } 3 \text { salarios } \\
\text { mínimos }\end{array}$ & 3,6 & & 1,7 & & 2,7 & & 4,3 & \\
\hline $\begin{array}{r}4 \text { a } 7 \text { salarios } \\
\text { mínimos }\end{array}$ & 3,9 & & 1,4 & & 3,1 & & 4,5 & \\
\hline
\end{tabular}




\begin{tabular}{|c|c|c|c|c|c|c|c|c|}
\hline \multicolumn{2}{|l|}{$\begin{array}{l}\text { Padre/madre } \\
\text { con más de un } \\
\text { hijo }\end{array}$} & \multicolumn{2}{|l|}{0,969} & \multirow[t]{3}{*}{0,304} & \multicolumn{3}{|c|}{0,363} & \multirow[t]{3}{*}{0,026} \\
\hline Sí & 3,7 & & 2,0 & & 2,8 & & 4,1 & \\
\hline No & 3,7 & & 1,8 & & 3,0 & & 4,7 & \\
\hline Religión & & 0,589 & & 0,029 & & 0,093 & & 0,248 \\
\hline Católico & 3,6 & & 2,2 & & 2,9 & & 4,0 & \\
\hline Protestante & 3,7 & & 1,6 & & 2,9 & & 4,5 & \\
\hline $\begin{array}{r}\text { Sin religión, pero } \\
\text { cree en Dios }\end{array}$ & 3,6 & & 2,0 & & 2,2 & & 4,4 & \\
\hline $\begin{array}{l}\text { Mayor factor } \\
\text { estresante }\end{array}$ & & 0,012 & & 0,026 & & 0,482 & & 0,946 \\
\hline $\begin{array}{l}\text { Aceptación/ } \\
\text { diagnóstico }\end{array}$ & 3,7 & & 2,0 & & 2,7 & & 4,3 & \\
\hline Internación & 4,3 & & 2,4 & & 3,3 & & 4,3 & \\
\hline Tratamiento & 3,6 & & 1,3 & & 2,7 & & 4,3 & \\
\hline $\begin{array}{r}\text { Recurrencia del } \\
\text { cáncer }\end{array}$ & 3,3 & & 1,4 & & 3,3 & & 4,0 & \\
\hline Falta de apoyo & 1,4 & & 1,4 & & 1,5 & & 4,3 & \\
\hline
\end{tabular}

También se notó que las personas mayores de 50 años obtuvieron el promedio más alto de "posición negativa ante Dios", a la vez que las del grupo de 17 a 29 años tuvieron el resultado más bajo. La dimensión de la ciudad reveló un mejor promedio de "revaluación negativa de Dios" para aquellos que viven en Recife. En contraste, el resultado más bajo fue para quienes viven en otros estados. Con respecto a la educación, los encuestados con escuela secundaria incompleta tuvieron el promedio más alto de "revaluación negativa del significado" y el promedio más bajo fue para aquellos que habían completado la educación superior. Para el estado civil, el mayor resultado en la dimensión "revaluación negativa del significado" se encontró entre los separados y el más bajo promedio fue entre los solteros. Finalmente, las personas sin religión pero que creen en Dios obtuvieron el promedio de CREN más alto para la variable de religión, a la vez que el promedio más bajo fue alcanzado por los católicos (Tabla 5).

Se encontró que el $71,8 \%$ de los encuestados, cuyos familiares fueron diagnosticados con cáncer hace máximo de 2 años, informaron que el momento más intenso de estrés durante todo este proceso de la enfermedad fue recibir y aceptar las noticias de la enfermedad. Sin embargo, para los pacientes con más tiempo desde el diagnóstico (más de 2 años), el 50\% de los miembros de la familia consideraron el tratamiento como el mayor estresante. 
Tabla 5. Asociación entre el CREN y sus dimensiones con las características sociodemográficas y personales de los familiares de pacientes hospitalizados en la sala de observación de oncología pediátrica del IMIP.

\begin{tabular}{|c|c|c|c|c|c|c|c|c|}
\hline \multirow[t]{2}{*}{ Variables } & \multicolumn{2}{|c|}{$\begin{array}{c}\text { Reevaluación } \\
\text { negativa de Dios }\end{array}$} & \multicolumn{2}{|c|}{$\begin{array}{l}\text { Posición negativa } \\
\text { ante Dios }\end{array}$} & \multicolumn{2}{|c|}{$\begin{array}{l}\text { Reevaluación } \\
\text { negativa del } \\
\text { significado }\end{array}$} & \multicolumn{2}{|c|}{ CREN } \\
\hline & Média & P-valor & Média & P-valor & Média & P-valor & Média & P-valor \\
\hline \multicolumn{9}{|l|}{ Edad } \\
\hline 17 a 29 años & 1,4 & 0,946 & 2,9 & $0,036^{*}$ & 2,3 & 0,898 & 1,9 & 0,434 \\
\hline 30 a 39 años & 1,4 & & 3,9 & & 2,6 & & 2,1 & \\
\hline 40 a 49 años & 1,4 & & 3,4 & & 2,5 & & 2,0 & \\
\hline$\geq 50$ años & 1,5 & & 4,5 & & 2,7 & & 2,4 & \\
\hline \multicolumn{9}{|l|}{ Ciudad } \\
\hline Recife & 1,8 & $0,010^{*}$ & 3,9 & 0,714 & 3,0 & 0,096 & 2,4 & 0,769 \\
\hline $\begin{array}{r}\text { Región Metropolitana de } \\
\text { Recife }\end{array}$ & 1,3 & & 3,3 & & 2,5 & & 2,0 & \\
\hline Interior de Pernambuco & 1,4 & & 3,6 & & 2,0 & & 1,9 & \\
\hline Otros estados & 1,0 & & 3,5 & & 2,2 & & 1,9 & \\
\hline \multicolumn{9}{|l|}{ Nivel Académico } \\
\hline Primaria Incompleta & 1,6 & 0,162 & 3,8 & 0,280 & 2,7 & $0,041 *$ & 2,2 & 0,061 \\
\hline Primaria Completa & 1,7 & & 4,5 & & 2,7 & & 2,5 & \\
\hline Secundaria Incompleta & 1,0 & & 3,7 & & 3,5 & & 2,2 & \\
\hline $\begin{array}{r}\text { Secundaria } \\
\text { Completa }\end{array}$ & 1,3 & & 3,2 & & 2,2 & & 1,9 & \\
\hline Superior Incompleta & 1,2 & & 2,2 & & 2,0 & & 1,5 & \\
\hline Superior Completa & 1,1 & & 3,4 & & 1,0 & & 1,6 & \\
\hline Posgraduación & 1,0 & & 3,0 & & 1,3 & & 1,5 & \\
\hline \multicolumn{9}{|l|}{ Estado Civil } \\
\hline Soltero & 1,2 & 0,887 & 3,1 & 0,458 & 1,9 & $0,046^{*}$ & 1,9 & 0,085 \\
\hline Casado & 1,4 & & 3,4 & & 2,0 & & 1,9 & \\
\hline Separado & 1,3 & & 4,1 & & 2,9 & & 2,2 & \\
\hline Relación estable & 1,5 & & 3,7 & & 2,7 & & 2,2 & \\
\hline \multicolumn{9}{|l|}{ Religión } \\
\hline Católico & 1,2 & 0,473 & 3,2 & 0,267 & 1,6 & 0,971 & 1,7 & $0,010^{*}$ \\
\hline Protestante & 1,5 & & 3,7 & & 2,8 & & 2,2 & \\
\hline $\begin{array}{r}\text { Sin religión, pero cree } \\
\text { en Dios }\end{array}$ & 1,4 & & 3,9 & & 2,8 & & 2,3 & \\
\hline
\end{tabular}




\section{Discusión}

Evaluar la CV de los familiares de pacientes con cáncer pediátrico, así como su religiosidad y espiritualidad como base para hacer frente a la situación estresante, es esencial para comprender mejor cómo estos aspectos interfieren en el proceso de bienestar, tratamiento y recuperación de la enfermedad ${ }^{(15)}$. Varios estudios, como el realizado en clínicas públicas en Minas Gerais (Brasil), señalan una relación positiva entre $\mathrm{R} / \mathrm{E}$ y mejores indicadores de salud, ya que las personas más espirituales tienen una mayor capacidad para enfrentar las circunstancias adversas de la vida ${ }^{(6)}$. Igualmente, será posible proporcionar mejores recursos para que los profesionales de la salud desarrollen una nueva visión de la importancia del enfoque de R/E, ya que, si se hace bien, brinda numerosos beneficios para la salud y la CV de los miembros de la familia y los pacientes ${ }^{(16)}$.

En el presente estudio, hubo un predominio de mujeres cuidadoras. Resultados similares se encontraron en otros estudios realizados en Brasil, en los estados de São Paulo, Rio Grande do Sul y Ceará. Esto puede explicarse por el hecho de que las mujeres, histórica y culturalmente, son responsables del cuidado de sus hijos, en todas sus etapas del ciclo de vida, convirtiéndose en cuidadoras de la familia y del hogar. En línea con estos resultados, se encontró que las madres predominaban en el cuidado infantil, como también se encontró en un estudio en Coímbra (Portugal) y Ciudad de México (México). La atribución de la maternidad otorga a las mujeres el papel de cuidadoras a quienes necesitan su atención, a nivel físico o psíquico, siendo uno de los componentes principales de la identidad femenina. A pesar de que están asumiendo nuevos roles fuera de la familia, la relación emocional con sus hijos sigue siendo fuerte, de modo que la abnegación materna a menudo regula la vida de una mujer ${ }^{(12,15,17)}$.

Otro dato importante por analizar es la edad promedio de los entrevistados. En el presente estudio, se evidenció un promedio de 35 años, lo que se verificó también en un estudio realizado en la Ciudad de México. Esta igualdad de promedios puede explicarse por la similitud sociodemográfica de los dos países. Así, los datos obtenidos se refieren al perfil de los sujetos en edad laboral, siendo la mayoría casados/en una relación estable, un hecho también visible en otros estudios con familiares de pacientes de oncología pediátrica, uno en Brasil y el otro en Canadá(4,9,17,18).

Es importante reforzar que una familia con una base estructural sólida tiene más facilidad para desarrollar acciones de unión, cuidado, amor y afrontamiento. Es por esta razón que las personas separadas exhibieron una peor capacidad de enfrentamiento al evaluar el estímulo estresante como fuerzas malignas y punitivas, como se ve en este trabajo, ya que es más difícil compartir deseos y obligaciones con excónyuges $^{(19)}$.

En este contexto, cuando se trabaja con la edad y la CV, la investigación ha demostrado que a mayor edad peor es la CV, así las personas mayores de 50 tenían el peor promedio. Según la literatura, el resultado anterior puede explicarse por las pérdidas y experiencias inherentes al proceso de envejecimiento, como los cambios en el cuerpo mismo, la muerte real de los seres queridos y amigos, el final de las relaciones laborales y los cambios en las relaciones sociales y familiares. Todos estos factores contribuyen a la disminución de la CV. En contraste con eses resultados, otro estudio realizado en México muestra que los jóvenes tienen los peores promedios. Esto se explica por el hecho de que ellos tienen más problemas financieros y mayores dificultades para manejar el dolor del paciente y continuar con sus actividades sociales ${ }^{(20)}$. 
Con respecto al grado de parentesco, se evidenció que los abuelos tenían los peores promedios, principalmente relacionados con factores psicológicos y de relación. También se observó que los hermanos/hermanas mostraron los mejores niveles de dominios emocionales, un hecho probablemente explicado por la poca exposición a las experiencias de duelo. También se descubrió que los padres tienen las tasas más altas de relaciones sociales en la $\mathrm{CV}$, ya que ocupan predominantemente el papel de proveedor, no participan tan activamente en el cuidado de sus hijos y, por lo tanto, no perjudican tanto sus relaciones personales ${ }^{(12,17,18,21)}$.

Los estudios también indican una asociación entre las variables estrés y afrontamiento con la edad, de modo que los mayores enfrentan situaciones adversas de manera diferente a las personas de otros grupos de edad. Se observó, que las personas mayores ya han sufrido tantas pérdidas físicas y emocionales que terminan recurriendo a la búsqueda de apoyo, principalmente religiosos/espirituales, como base para hacer frente. Sin embargo, esta búsqueda es tan intensa que a menudo terminan rindiendo toda resolución del problema al poder de lo divino, ya que están tan agotados debido al proceso de envejecimiento en sí y sus consecuencias. Por lo tanto, al evaluar el afrontamiento de R/E a través del afrontamiento de esta investigación, se notó que las personas mayores de 50 años presentaban una mayor posición negativa hacia Dios, transfiriendo toda la responsabilidad de curar la enfermedad, sin ningún esfuerzo ${ }^{(22,23)}$.

Como se indicó anteriormente, los participantes en esta investigación están predominantemente en edad laboral y, por lo tanto, se espera que la mayoría trabaje. Similar a estos datos, en una encuesta realizada en el estado brasileño de Rio Grande do Sul, la mayoría de los miembros de la familia también estaban en edad productiva, de modo que el 61,7\% trabajaba. Por el contrario, un estudio en Granada (España) mostró una prevalencia de participantes que no trabajan. Sin embargo, en el estudio español, la edad promedio es de 51,8 años, lo que muestra un predominio de personas que ya están en la etapa final de la edad productiva o ya están retiradas ${ }^{(15,24)}$.

En esta investigación se encontró que los cuidadores que trabajan tienen una mejor $\mathrm{CV}$, especialmente con respecto a la sensación de seguridad física, ambiental y financiera. La razón de esto se puede encontrar en el hecho de que las personas que tienen trabajo tienen una mejor estabilidad financiera $y$, por lo tanto, pueden satisfacer mejor sus necesidades en comparación con las personas que no están trabajando $^{(25)}$.

La mayoría de los familiares entrevistados tienen un trabajo, con un ingreso familiar de 1 a 3 salarios mínimos. Esto es un reflejo del perfil sociodemográfico de los individuos que utilizan el servicio de salud pública en Brasil, cuya mayoría $(85,1 \%)$ está compuesta por personas con un ingreso mensual de hasta 3 salarios mínimos, según un estudio realizado en 2015 que caracteriza el perfil socioeconómico de los usuarios del Sistema Único de Salud (SUS). Además, dicho este demostró el perfil de los usuarios en el Noreste de Brasil, misma región donde se realizó la recolección de datos para el presente estudio, en el cual 95\% de las personas formaban parte del grupo con ingresos correspondientes hasta de 3 salarios mínimos, que fue consistente con los resultados obtenidos en esta encuesta de $2015^{(26)}$.

Según el Instituto de Investigación Económica Aplicada(IPEA), existen componentes esenciales para el bienestar familiar que dependen directamente de los ingresos mensuales, lo que influye en la capacidad de comprar productos y servicios, proporcionando una mejor salud, condiciones sanitarias, transporte y tiempo recreativo, hecho o información 
que se corrobora con los resultados encontrados en aquellos con mayores recursos financieros, los cuales tienen mejor CV. Además, también se verificó que las personas que viven con menos de un salario mínimo tienen una mejor CV en relación con los entrevistados con un ingreso salarial entre 1 y 3 . Esto puede ser justificado, en parte, por los miembros del grupo "menos de 1 salario mínimo" tienen expectativas de que pueden cumplir de acuerdo con su condición de vida, diferente de aquellos entre 1 y 3 salarios. Estos últimos, probablemente, tienen otros anhelos como la realización de deseos y estatus social muchas veces todavía no logrados, resultando en una disminución de la satisfacción con la vida resultando en una disminución de la satisfacción con la vida ${ }^{(27)}$.

Es posible percibir todavía la influencia de las cuestiones financieras en el modelo de afrontamiento religioso/espiritual adoptado. El bajo ingreso familiar resultó en un mayor uso del CREP, especialmente con respecto a la búsqueda de apoyo espiritual, representando una fuente importante de fortaleza y esperanza ante las dificultades. En oposición a estos datos, un estudio realizado en una clínica pública en Minas Gerais encontró que los ingresos mensuales desfavorecidos generan un mayor uso de CREN, porque, según sus datos, el ingreso está fuertemente asociado con el ajuste psicológico de la familia en su conjunto, de modo que además de los aspectos humanos involucrados en el proceso de salud/enfermedad, las variables vinculadas al contexto financiero y material de la vida de las personas también proporcionan un sustrato para el apoyo emocional y el manejo del cáncer ${ }^{(6)}$.

Autores declaran que un buen nivel de educación garantiza mayores posibilidades para aprender diferentes formas de lidiar con los problemas. Además, estos individuos desarrollan más facilidad en el uso de estrategias cognitivas y conductuales, lo que les permite la capacidad de descentralizar problemas emocionales y participar en actividades productivas, incluida la búsqueda de apoyo social y espiritual. En congruencia con estos datos, se observó en los resultados de la presente investigación que cuanto mayor es la escolaridad, mejor es el CRE ${ }^{(28,29)}$.

$\mathrm{Al}$ asociar CV y lugar de residencia, el mejor promedio es notable para los residentes de la capital en comparación con los que vivían más lejos, como en el campo u otros estados. Esto también se observó en una encuesta realizada en Recife y otro en el sudeste de Brasil, a lo que demostraron consecuencias negativas para la rutina familiar de los participantes que vivieron en otros estados, con impacto directo en varios aspectos psicosociales haciendo que la familia se aleje de su entorno de vida en busca de mejores recursos de tratamiento ${ }^{(18,30)}$.

También se observó que los entrevistados que viven en la capital tienen mayores sentimientos negativos en relación a si mismo, como culpa, revuelta e desamparo divino, lo que caracteriza un CREN. Esto puede entenderse como consecuencia de los problemas de la vida urbana, tales como estrés, ansiedad, irritabilidad, intolerancia e inseguridad. Sin embargo, no se encontraron estudios que evaluaron esta variable, lo que dificultó la comparación del resultado obtenido ${ }^{(31)}$.

Asimismo, se observó una relación entre tener más de un hijo y el afrontamiento positivo. Los padres que tienen un solo hijo pueden deshacerse del estímulo estresante de manera más eficiente, sin negar su existencia, a través de la conexión con la $\mathrm{R} / \mathrm{E}$, presentando, así, mejor CRE. Esto también se encontró en el estudio realizado en la ciudad de Málaga (España) y otro en un centro de referencia nacional para el tratamiento del cáncer infantil, los cuales afirmaron que el hecho de que haya otros niños involucrados aumenta la carga de culpa que sienten los padres por centralizar la atención, distanciándose de los niños $\operatorname{sanos}^{(18,32)}$. 
Se observó en el presente estudio que los participantes religiosos presentan un mejor CRE en comparación con aquellos que declararon que no tenían religión. Esto puede explicarse por la literatura, la cual afirma que las personas con prácticas religiosas buscan apoyo en estas actividades para lidiar con las dificultades ${ }^{(33)}$. Por lo tanto, individuos sin religión, incluso si espiritualizados, tienen un mayor uso de CREN, ya que la ausencia de creencias y prácticas espirituales no les permite sentir la esperanza, equilibrio y empoderamiento ofrecido por la $\mathrm{R} / \mathrm{E}$, proporcionando voluntad de luchar por la vida y serenidad para aceptar la enfermedad, como también se vio en los resultados de nuestra investigación ${ }^{(6)}$.

De acuerdo con la literatura, existe una asociación favorable entre $R / E$, salud y $\mathrm{CV}$, porque las prácticas espirituales evocan emociones elevadoras ${ }^{(15)}$. El estudio en Minas Gerais incluso abordó la contribución del CRE como un factor mitigante para las complicaciones. En él, el 53\% de las personas asistieron a la institución religiosa/espiritual una o más veces a la semana y consideraron estos momentos esenciales para alcanzar la plenitud. Estas últimas presentaron buenos niveles de CV. En esta encuesta, $12 \%$ de los entrevistados informaron una rara frecuencia a la iglesia y otras instituciones espirituales, presentando los peores índices de $\mathrm{CV}^{(6)}$. Sin embargo, al analizar los datos obtenidos en nuestra investigación, se notó que los mejores promedios de CV eran para personas cuya frecuencia religiosa/espiritual era rara, quedando para el grupo con una regularidad de 1 o más veces por semana la segunda posición. Esta divergencia de resultados puede explicarse por el reducido número de sujetos que raramente se dedican a las prácticas religiosas, compuesto por solo 5 personas, lo que puede haber dificultado el análisis.

Es posible observar diferentes niveles de $\mathrm{CV}$ de acuerdo con el mayor factor de estrés durante el proceso de la enfermedad. Los miembros de la familia que consideraron la recurrencia del cáncer y la aceptación del diagnóstico como el peor momento para enfrentarse, consiguieron una mejor $\mathrm{CV}$, lo que se puede explicar porque es en estos momentos inesperados que existe una mayor movilización socioafectiva por parte de familiares, amigos y conocidos ${ }^{(18,34)}$. En cuanto a los que dijeron que la hospitalización fue el mayor estrés experimentado, tuvieron una menor CV porque la hospitalización del niño interfiere directamente con la vida del cuidador, causando un mayor aislamiento social y cambios en la rutina matrimonial y familiar ${ }^{(35,36)}$. Los estudios realizados en la región sureste de Brasil y en la Ciudad de México indicaron lo mismo, lo que colabora con nuestros hallazgos ${ }^{(17,30)}$.

En este contexto de factores estresantes, la perspectiva acerca del CREP puede analizarse de diferentes maneras dependiendo de cuál momento de tensión fue considerado más relevante por los familiares. Los participantes que consideraron la hospitalización como el peor factor, tuvieron más oportunidades para reflexiones sobre la espiritualidad, la vida y sus propias actitudes, debido a la interrupción de la rutina. Para convivir mejor con la hospitalización, las personas tienden a reorganizar aspectos de la vida intra y extrahospitalaria, recurriendo muchas veces a la $R / E$, lo que permite el desarrollo de estrategias adaptativas para mantener el equilibrio emocional. Por estas razones, la presente investigación indicó, para familiares incluidos en este grupo, los mejores afrontamiento de $\mathrm{R} / \mathrm{E}$ relacionado con la transformación de si mismo y la búsqueda de ayuda espiritual ${ }^{(9,19,37)}$.

Así como el presente estudio, la revisión de la literatura y la revisión sistemática susodichos también demostraron una relación significativa y directa entre el afrontamiento religioso/espiritual y la CV de los entrevistados. Se observó que los 
familiares y los pacientes utilizan predominantemente la $\mathrm{R} / \mathrm{E}$ como una forma de enfrentamiento de la enfermedad y esto tiene un impacto positivo en la $\mathrm{CV}$, resultando en sentimientos de optimismo y amparo, mejor salud mental, más tranquilidad en tiempos de adversidad y mayor aceptación de sus propios límites ${ }^{(3,9)}$.

\section{Conclusiones}

El estudio muestra que, para el público evaluado, la espiritualidad es un factor importante para el enfrentamiento de situaciones estresantes como también proporciona una mejor $\mathrm{CV}$ a los familiares de pacientes con cáncer pediátrico, corroborando con otros que demuestran que la religiosidad y la espiritualidad son factores relevantes para enfrentar las adversidades a las cuáles están expuestos los pacientes y sus familiares, vinculándolas con el suministro de apoyo emocional, instrumental e informativo.

El presente estudio también demostró que los pacientes quieren que su espiritualidad sea abordada por profesionales médicos, trayendo así, del punto de vista de las praxis médicas, la necesidad del especialista en oncología pediátrica considerar los diversos beneficios proporcionados por el uso de estrategias positivas de CRE, como por ejemplo: mayor adherencia al tratamiento, facilitación del acceso a las redes de apoyo y la integración social, producción de sentido/propósito de la vida, esperanza y reducción de los síntomas depresivos.

Desde el punto de vista científico, existe una clara necesidad de más investigación sobre el tema y, en especial, estudios que también involucren niños, adolescentes y profesionales de la salud. Teniendo en vista la complejidad de la expresión espiritual y religiosa en el mundo, es indudable el valor de las futuras contribuciones al campo de la salud.

\section{Referencias bibliográficas}

1. Moreira-Almeida A, Stroppa A. Espiritualidade e saúde mental: o que as evidências mostram? Revista Debates em Psiquiatria 2012; 2: 34-41.

2. Moreira-Almeida A, Lucchetti G. Panorama das pesquisas em ciência, saúde e espiritualidade. Ciência e Cultura; 68: 54-7. doi: 10.21800/2317-66602016000100016

3. Meireles CB, Maia LC, Miná VAL, Novais MSMC, Peixoto JAC, Cartaxo MABS, et al. Influence of spirituality in pediatric cancer management: a systematic review. Inter Arch Med 2015; 8: 1-13. doi: 10.3823/1634

4. Araújo Alves D, Silva LG, Araújo Delmondes G, Lemos ICS, Kerntopf MR, Albuquerque GA. Cuidador de Criança com Câncer: Religiosidade e Espiritualidade como Mecanismos de Enfrentamento. Revista Cuidarte 2016; 7: 1318-24. doi: 10.15649/cuidarte.v7i2.336

5. Angelo M. Ouvindo a voz da família: narrativas sobre o sofrimento e espiritualidade. O mundo da Saúde 2010; 34: 437-43. doi: 10.15343/0104-7809.20104437443

6. Mesquita AC, Chaves ECL, Avelino CCV, Nogueira DA, Panzini RG, Carvalho EC. A utilização do enfrentamento religioso/espiritual por pacientes com câncer em tratamento quimioterápico. Rev Lat Am Enfermagem 2013; 21: 539-45. doi: 10.1590/S010411692013000200010

7. Garanito MP, Cury MRG. A espiritualidade na prática pediátrica. Revista Bioética 2016; 24: 49-53. doi: 10.1590/1983-80422016241105 
8. Velasco C, Bengoechea C, López-Ibor B. Afrontamiento y bienestar psicológico en padres de niños y adolescentes con cáncer durante el tratamiento. Psiconcología 2019; 16: 227-49. doi: 10.5209/psic.65589

9. Nicholas DB, Barrera M, Granek L, D’Agostino NM, Shaheed J, Beaune L, et al. Parental spirituality in life-threatening pediatric câncer. J Psychosoc Oncol 2017; 35: 323-34. doi: 10.1080/07347332.2017.1292573

10. Damiano RF, Costa LA, Viana MTSA, Moreira-Almeida A, Lucchetti ALG, Lucchetti G. Brazilian scientific articles on "Spirituality, Religion and Health". Arch Clinl Psychiatr 2016;43:11-6. doi: 10.1590/0101-60830000000073

11. Koenig HG, King DE, Carson. Handbook of Religion and Health. 2nd ed. Oxford. Oxford University Press 2012

12. Batalha LMC, Fernandes AM, Campos C. Qualidade de vida em crianças com câncer: concordância entre crianças e pais. Esc. Anna Nery Rev de Enferm 2015; 19: 292-6. doi: 10.5935/1414-8145.20150039

13. Lucchetti G, Lucchetti ALG, Vallada H. Measuring spirituality and religiosity in clinical research: a systematic review of instruments available in the Portuguese language. São Paulo Med J 2013; 131: 112-22. doi: 10.1590/S1516-31802013000100022

14. Pedroso B, Pilatti LA, Gutierrez GL, Picinin CT. Cálculo dos escores e estatística descritiva do WHOQOL-bref através do Microsoft Excel. Revista Brasileira de Qualidade de Vida 2010; 2: 31-36. doi: 10.3895/S2175-08582010000100004

15. Moura Fetsch CF, Portella MP, Kirchner RM, Gomes JS, Benetti ERR, Stumm EMF. Estratégias de afrontamiento entre familiares de pacientes oncológicos. Revista Brasileira de Cancerologia 2016; 62: 17-25. doi: 10.32635/2176-9745.RBC.2016v62n1.175

16. Negreiros RV, Sá Furtado I, Vasconcelos CRP, Souza LSB, Vilar MMG, Alves RF. A Importância do apoio familiar para a efetividade no tratamento do câncer infantil: uma vivência hospitalar. Revista Saude \& Ciencia online 2017; 6: 57-64.

17. Pardo XM, Cárdenas SJ, Venegas JM. Variables que predicen la aparición de sobrecarga en cuidadores primarios informales de niños con cáncer. Psicooncología 2015; 12: 67-86. doi: 10.5209/rev_PSIC.2015.v12.n1.48905

18. Beltrão MRLR, Vasconcelos MGL, Pontes CM, Albuquerque MC. Câncer Infantil: percepções maternas e estratégias de enfrentamento frente ao diagnóstico. Jornal de Pediatria 2007; 83: 562-566. doi: 10.1590/S0021-75572007000800014

19. Figueiredo T, Silva AP, Silva RMR, Jesus Silva J, Oliveira e Silva CS, Alcântara DDF, et al. Como posso ajudar? Sentimentos e experiências do familiar cuidador de pacientes oncológicos. Arquivos Brasileiros de Ciências da Saúde 2017; 42: 34-9. doi: 10.7322/ abcshs.v42i1.947

20. Vázquez OG, Castillo ER, Huertas LA, García AM, Ponce JLA, Manzanilla EO, et al. Guía de práctica clínica para la atención psico-oncológica del cuidador primario informal de pacientes con cáncer. Psicooncología 2015; 12: 87-104. doi: 10.5209/rev_PSIC.2015.v12.n1.48906

21. Borges EL, Franceschini J, Costa LHD, Fernandes ALG, Jamnik S, Santoro IL. Sobrecarga do cuidador familiar: a sobrecarga de cuidar de pacientes com câncer de pulmão, de acordo com o estágio do câncer e a qualidade de vida do paciente. J Bras Pneumo 2017; 43:18-23. doi: s1806-37562016000000177

22. Cocentino JMB, Camargo Viana T. A Velhice e a Morte: reflexões sobre o processo de luto. Rev Bras Geriatr Gerontol 2011; 14: 591-600. doi: 10.1590/S1809-98232011000300018

23. Santos Ribeiro M, Silva Borges M, Araújo TCCF, Santos Souza MC. Estratégias de enfrentamento de idosos frente ao envelhecimento e à morte: revisão integrativa. Rev Bras Geriatr Gerontol 2017; 20: 880-8. doi: 10.1590/1981-22562017020.170083 
24. Romero V, Cruzado JA. Duelo, ansiedad y depresión en familiares de pacientes en una unidad de cuidados paliativos a los dos meses de la pérdida. Psicooncología 2016; 13: 23-37. doi: 10.5209/rev_PSIC.2016.v13.n1.52485

25. Campara JP, Vieira KM, Potrich ACG. Satisfação Global de Vida e Bem-estar Financeiro: desvendando a percepção de beneficiários do Programa Bolsa Família. Revista de Administração Pública 2017; 51: 182-200. doi: 10.1590/0034-7612156168

26. Guibu IA, Moraes JC, Guerra Junior AA, Costa EA, Assis Acurcio F, Costa KS, et al. Características principais dos usuários dos serviços de atenção primária à saúde no Brasil. Revista de Saúde Pública 2017; 51: 1-13. doi: 10.11606/S1518-8787.2017051007070

27. Junior JCC, Cunha AS. Planejamento e Avaliação de Políticas Públicas. Brasil. Instituto de Pesquisa Econômica Aplicada (IPEA) 2015.

28. Sousa Certal Martins C. Impacto do suporte social e dos estilos de afrontamiento sobre a percepção subjetiva de bem-estar e qualidade de vida em doentes com lúpus. Psicologia. pt: o portal dos psicólogos 2007; p. 1-20. [Acceso 10/08/2020] Disponible en: https:// www.psicologia.pt/artigos/textos/A0339.pdf

29. Noriega JAV, Albuquerque FJB, Alvarez JFL, Pimentel CE. Afrontamiento em uma população do nordeste brasileiro. Avaliação Psicológica 2003; 2(1): 17-27. [Acceso 10/08/2020] Disponible en: http://pepsic.bvsalud.org/scielo.php?script= sci arttext\&pid $=$ S1677-04712003000100003\&lng=pt\&nrm $=$ iso

30. Arruda-Colli MNF, Lima RAG, Perina EM, Santos MA. A recidiva do câncer pediátrico: um estudo sobre a experiência materna. Psicologia USP 2016; 27: 307-14. doi: 10.1590/0103-656420140078

31. Ribeiro H, Vargas HC. Urbanização, globalização e saúde. Revista USP 2015; 107: 13-26. doi: 10.11606/issn.2316-9036.v0i107p13-26

32. Melguizo-Garín A, Martos-Méndez MJ, Hombrados-Mendieta, I. Influencia del apoyo social sobre el estrés y la satisfacción vital en padres de niños con cáncer desde una perspectiva multidimensional. Psicooncología 2019; 16: 25-42. doi: 10.5209/PSIC.63646.

33. Brasileiro TOZ, Souza VHS, Oliveira Prado AA, Lima RS, Nogueira DA, Cassia Lopes Chaves E. Bem-estar espiritual e afrontamiento religioso/espiritual em pessoas com insuficiência renal crônica. Avances en Enfermería 2017; 35: 159-70. doi: 10.15446/ av.enferm.v35n2.60359

34. Arruda-Colli MNF, Santos MA. Aspectos psicológicos da recidiva em Oncologia Pediátrica: uma revisão integrativa. Arquivos Brasileiros de Psicologia 2015; 67: 75-93. [Acceso 10/08/2020] Disponible en: http://pepsic.bvsalud.org/scielo.php?script=sci_ arttext\&pid $=$ S1809-52672015000300007\&lng=pt\&nrm $=$ iso

35. Sales CA, de Matos PCB, de Mendonça DPR, Marcon SS. Cuidar de um familiar com câncer: o impacto no cotidiano de vida do cuidador. Revista Eletrônica de Enfermagem 2010; 12: 616-21. doi: 10.5216/ree.v12i4.12160

36. Mensorio MS, Kohlsdorf M, Costa Junior AL. Cuidadores de crianças e adolescentes com leucemia: análise de estratégias de enfrentamento. Psicologia em Revista 2009; 15: 15876. [Acceso 10/08/2020] Disponible en: http://pepsic.bvsalud.org/scielo.php?script=sci arttext\&pid $=$ S1677-11682009000100010\&lng=pt\&nrm=iso

37. Peres GM, Lopes AMP. Acompanhamento de pacientes internados e processos de humanização em hospitais gerais. Psicologia Hospitalar 2012; 10: 17-41. [Acceso 10/08/2020]. Disponible en: http://pepsic.bvsalud.org/scielo.php?script=sci arttext\&pid $=$ S1677-74092012000100003\&lng=pt\&nrm $=$ iso 